OPEN ACCESS

Edited by:

Megan Laura McCain,

University of Southern California,

United States

Reviewed by:

Anthony Brown,

The Ohio State University,

United States

Vincent Timmerman,

University of Antwerp, Belgium

Florian Barthelemy,

University of California, Los Angeles,

United States

*Correspondence:

Bruce R. Conklin

bconklin@gladstone.ucsf.edu

Luke M. Judge

luke.judge@ucsf.edu

Specialty section:

This article was submitted to

Stem Cell Research,

a section of the journal

Frontiers in Cell and Developmental

Biology

Received: 09 June 2021

Accepted: 22 July 2021

Published: 16 August 2021

Citation:

Feliciano CM, Wu K, Watry HL,

Marley CBE, Ramadoss GN,

Ghanim HY, Liu AZ, Zholudeva LV,

McDevitt TC, Saporta MA, Conklin BR and Judge LM (2021) Allele-Specific Gene Editing Rescues Pathology in a

Human Model

of Charcot-Marie-Tooth Disease

Type 2E.

Front. Cell Dev. Biol. 9:723023.

doi: 10.3389/fcell.2021.723023

\title{
Allele-Specific Gene Editing Rescues Pathology in a Human Model of Charcot-Marie-Tooth Disease Type 2E
}

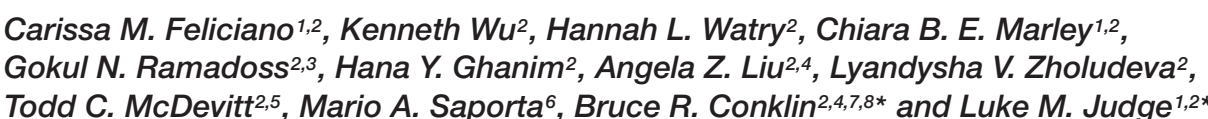

${ }^{1}$ Department of Pediatrics, University of California, San Francisco, San Francisco, CA, United States, ${ }^{2}$ Gladstone Institutes, San Francisco, CA, United States, ${ }^{3}$ Biomedical Sciences Ph.D. Program, University of California, San Francisco, San Francisco, CA, United States, ${ }^{4}$ Department of Ophthalmology, University of California, San Francisco, San Francisco, CA, United States, ${ }^{5}$ Department of Bioengineering and Therapeutic Sciences, University of California, San Francisco, San Francisco, CA, United States, ${ }^{6}$ Department of Neurology, Miller School of Medicine, University of Miami, Miami, FL, United States, ${ }^{7}$ Department of Medicine, University of California, San Francisco, San Francisco, CA, United States,

${ }^{8}$ Innovative Genomics Institute, Berkeley, CA, United States

Many neuromuscular disorders are caused by dominant missense mutations that lead to dominant-negative or gain-of-function pathology. This category of disease is challenging to address via drug treatment or gene augmentation therapy because these strategies may not eliminate the effects of the mutant protein or RNA. Thus, effective treatments are severely lacking for these dominant diseases, which often cause severe disability or death. The targeted inactivation of dominant disease alleles by gene editing is a promising approach with the potential to completely remove the cause of pathology with a single treatment. Here, we demonstrate that allele-specific CRISPR gene editing in a human model of axonal Charcot-Marie-Tooth (CMT) disease rescues pathology caused by a dominant missense mutation in the neurofilament light chain gene (NEFL, CMT type 2E). We utilized a rapid and efficient method for generating spinal motor neurons from human induced pluripotent stem cells (iPSCs) derived from a patient with CMT2E. Diseased motor neurons recapitulated known pathologic phenotypes at early time points of differentiation, including aberrant accumulation of neurofilament light chain protein in neuronal cell bodies. We selectively inactivated the disease NEFL allele in patient iPSCs using Cas9 enzymes to introduce a frameshift at the pathogenic N98S mutation. Motor neurons carrying this allele-specific frameshift demonstrated an amelioration of the disease phenotype comparable to that seen in an isogenic control with precise correction of the mutation. Our results validate allele-specific gene editing as a therapeutic approach for CMT2E and as a promising strategy to silence dominant mutations in any gene for which heterozygous loss-of-function is well tolerated. This highlights the potential for gene editing as a therapy for currently untreatable dominant neurologic diseases.

Keywords: induced pluripotent stem cells, Charcot-Marie-Tooth, neuropathy, motor neurons, dominant, CRISPRCas9, gene editing 


\section{INTRODUCTION}

Charcot-Marie-Tooth disease (CMT), also known as hereditary motor and sensory neuropathy, is one of the most common inherited neurological disorders, affecting approximately 1 in 2,500 people (Reilly et al., 2011). Patients present with muscle weakness and variable sensory symptoms in the distal limbs, which typically progress in a length-dependent manner, affecting the most distal limbs first. CMT can be further classified by the predominantly affected cell type, by clinical electrophysiological testing, and by underlying genetic cause (Rossor et al., 2015). The demyelinating forms of the disease (CMT1, autosomal dominant and CMT4, autosomal recessive) primarily affect Schwann cells and are characterized by a decrease in nerve conduction velocity (Brennan et al., 2015). The axonal forms of the disease (CMT2, dominant and recessive) are characterized by pathology intrinsic to motor and sensory neurons (Luigetti et al., 2016). The genetic causes of CMT are remarkably diverse, with causative mutations in over 80 genes, and a diverse set of individual mutations reported for many of those genes (DiVincenzo et al., 2014; Timmerman et al., 2014; Pareyson et al., 2017).

Axonal CMT is predominantly caused by autosomal dominant missense mutations, which occur in more than 20 genes with diverse functions (Rossor et al., 2015; Pareyson et al., 2017). A notable example is CMT2E, caused by mutations in the NEFL gene, which encodes the neurofilament light chain (NFL) protein. Neurofilaments are neuron-specific intermediate filaments composed of multiple subunits, including NF-L, that contribute to the radial growth of axons and the maintenance of axonal diameter and function (Szaro and Strong, 2010; Yuan et al., 2017). A multitude of missense mutations throughout the NEFL coding sequence cause autosomal dominant CMT2E (Stone et al., 2019). By contrast, loss-of-function mutations in NEFL are recessive, and only cause CMT when homozygousthe heterozygous carriers are neurologically normal (Abe et al., 2009; Yum et al., 2009; Fu and Yuan, 2018). These loss-offunction mutations demonstrate that a single functional NEFL allele is adequate for normal neurological function and suggest that dominant missense mutations in NEFL act via dominantnegative or pathologic gain-of-function mechanisms. The N98S mutation is a prime example of the latter, as a single mutant allele leads to the aberrant accumulation of neurofilaments in neuronal cell bodies and causes a severe and early onset neuropathy in humans and mice (Jordanova et al., 2003; Adebola et al., 2015; Saporta et al., 2015; Zhao et al., 2017; Lancaster et al., 2018).

There is currently no effective treatment for any form of CMT. Viral gene replacement therapy for the motor neuron disease spinal muscular atrophy (SMA) has shown great promise and could represent a viable strategy for the rare recessive forms of CMT2 (Mendell et al., 2017). However, dominant CMT2 likely requires an alternate approach as simply increasing expression of a wild-type gene may not overcome the pathologic effects of the mutant allele, which will continue to be expressed. Advances in gene-editing technologies create an opportunity to develop therapies that interrupt the pathologic processes causing dominant CMT2, and other dominant neurodegenerative disorders, at its genetic source (Porteus, 2019). Since people with a single functional NEFL allele have normal neurological function, we reasoned that selective inactivation of dominant missense mutations in NEFL via CRISPR-Cas9 gene editing would be therapeutic. We tested this hypothesis using an established human induced pluripotent stem cell (iPSC) model of CMT2E with the N98S mutation in NEFL (Saporta et al., 2015).

Here, we report a gene-editing strategy specific for the N98S mutation that efficiently and selectively reduced expression of the mutant allele in CMT2E motor neurons. Edited neurons displayed phenotypes that were similar to unrelated and isogenic controls, indicating that the editing strategy effectively rescued disease-associated pathology. This is the first reported application of gene editing for axonal CMT. It provides proof-of-principle for strategies that could be applied to other dominant-negative inherited neurodegenerative diseases.

\section{MATERIALS AND METHODS}

\section{iPSC Lines and Culture}

The healthy control iPSC line known as wild type C (WTC) used in these studies is an extensively characterized line from a healthy individual (Kreitzer et al., 2013) that is the parental line for the Allen Institute Cell Collection ${ }^{1}$. A CMT2E iPSC line derived from a female individual with the N98S mutation in NEFL causing childhood onset CMT has been previously characterized (Saporta et al., 2015; Maciel et al., 2020). The N98S mutation occurs in the CoillA domain of the neurofilament light chain (NF-L) protein at the 98th amino acid in both human and mouse orthologs. The mutation has also been referred to in the literature as N97S, likely due to variable inclusion of the start codon in the numbering system. iPSCs were cultured in Stemfit Basic02 (Ajinomoto) on plates coated with matrigel (Corning, 356231 ) at $37^{\circ} \mathrm{C}, 5 \% \mathrm{CO}_{2}, 85 \%$ humidity. iPSC cultures were passaged every 3-4 days with Accutase (Stem Cell Technologies, 07920) or ReLeSR (Stem Cell Technologies, 05872) into Stemfit supplemented with $10 \mu \mathrm{m}$ Y-27632 (SelleckChem, S1049).

\section{Generating Transgenic iPSC Lines With Inducible Transcription Factors}

The iPSCs were engineered to contain a $13-\mathrm{kb}$ vector containing the human transcription factors NGN2, ISL1, and LHX3 (hNIL) under the control of doxycycline (Fernandopulle et al., 2018). To knock-in the hNIL construct into safe harbor loci, $1 \times 10^{6}$ cells were added to $100 \mu \mathrm{L}$ P3 buffer with $6 \mu \mathrm{g}$ of the hNIL donor plasmid, and $3 \mu \mathrm{g}$ of each paired TALEN (Addgene, 59025/59026 or 62196/62197, respectively) targeting either AAVS1 or CLYBL (Cerbini et al., 2015). Cells were transfected using the P3 Primary Cell 4D-Nucleofector X Kit L (Lonza, V4XP-3024) with pulse code DS138. After nucleofection, cells incubated for $5 \mathrm{~min}$ at room temperature and were then plated in Stemfit media with $10-\mu \mathrm{M}$ Y-27632 in a serial dilution into five wells of a six-well plate. Non-transfected cells were plated in the sixth well. After 2-3 days post-nucleofection, cells

\footnotetext{
${ }^{1}$ http://www.allencell.org/
} 
were fed with Stemfit with Puromycin or G418 (depending on which donor vector was used) and 10- $\mu \mathrm{M}$ Y-27632. Cells were kept on antibiotic selection until all cells in the control wells were dead and only red fluorescent colonies remained in the experimental wells. Fluorescent, antibiotic-resistant colonies were then manually picked and transferred to individual wells of a 48-well plate.

DNA from each clone was extracted using the DNeasy Blood and Tissue Kit (Qiagen, 69506). PCRs were performed with primers spanning the $5^{\prime}$ and $3^{\prime}$ junctions of the integration, with one primer annealing within the construct and the other outside of the corresponding homology arm. A third PCR amplifying the intact safe harbor locus was performed to determine whether the clone harbored a heterozygous or homozygous insertion. PCR primer sequences are listed in Supplementary Table 1. For the CMT2E line, we inserted hNIL in the AAVS1 locus (Supplementary Figure 1) and obtained a previously published WTC line with hNIL inserted in the AAVS1 locus as a control (Shi et al., 2018). We also independently inserted hNIL in the CLYBL locus of the same parental WTC line (Supplementary Figure 1). When performing neuronal differentiation, we found equivalent results with the AAVS1 and CLYBL versions of the WT line. For comparison with the N98S-hNIL-AAVS1 line, we used WTC-hNIL-AAVS1 for all experiments, with the exception of manual image analysis shown in Figure 2, in which case we used the WTC-hNILCLYBL line.

\section{CRISPR/Cas9 Gene-Editing Analysis}

To prepare the Sp.HiFi Cas9 ribonucleoprotein (RNP) complex, 240 pmol of guide RNA (IDT) was mixed with 120 pmol of Hi-Fi SpCas9 protein (Macrolab) and incubated for $30 \mathrm{~min}$ at room temperature. For the Sa.KKH Cas9 RNP complex, 240 pmol of guide RNA (Synthego) was mixed with 120 pmol of Sa.KKH Cas9 protein (Macrolab). The gRNA sequences are listed in Supplementary Table 2. After dissociation with Accutase, $3.5 \times 10^{5}$ cells were resuspended in P3 buffer and mixed with the RNP complex. The iPSCs were transfected using the P3 Primary Cell 4D-Nucleofector X Kit S (Lonza, V4XP3032) with pulse code DS138. After nucleofection, cells were incubated for $5 \mathrm{~min}$ at room temperature and then plated in Stemfit with 10- $\mu$ M Y-27632 (SelleckChem). Genomic DNA was extracted from edited and unedited cells 3 days postnucleofection using the DNeasy Blood and Tissue Kit (Qiagen). PCRs were performed with primers spanning the gRNA binding site (Supplementary Table 1), and the PCR products were sequenced. The indel frequency at the cut site was determined using Synthego ICE software, which compares the Sanger sequencing traces from the edited and unedited populations (Supplementary Figure 2).

\section{Isolation of Clonal Edited Lines}

To generate the N98S-fs line, $3.5 \times 10^{5}$ cells were transfected with RNP as described above. After nucleofection, $0.1 \times 10^{5}$ cells were seeded onto a $10-\mathrm{cm}$ plate in Stemfit with $10-\mu \mathrm{M}$ Y-27632. After 5 days post-nucleofection, colonies were manually picked and transferred to individual wells of a 48-well plate. DNA was extracted from the cells using QuickExtract DNA Extract Solution (Lucigen, QE9050). Clones were genotyped by PCR and Sanger Sequencing (Supplementary Figure 3B).

To generate the N98S-corrected line, $3.5 \times 10^{5}$ cells were nucleofected with RNP and 50 pmol of donor DNA (IDT, Supplementary Table 1). To enrich for the homology directed repair (HDR) event and isolate a clone with the desired edit, allele-specific droplet-digital PCR (ddPCR) and sib-selection were performed (Miyaoka et al., 2014; Supplementary Figure 3C). After nucleofection, cells were plated at $2.5 \times 10^{3}$ cells per well in a 96-well plate. DNA was extracted from the cells using QuickExtract and analyzed by allele-specific ddPCR to measure HDR efficiency. The well with the highest HDR efficiency was replated at 10 cells per well in a 96-well plate for an additional round of enrichment. From this round, the well with the highest HDR efficiency $(\sim 11.4 \%)$ was replated sparsely into a $10-\mathrm{cm}$ plate for manual clone picking. The clones were genotyped by allele-specific ddPCR and further validated by PCR and Sanger Sequencing (Supplementary Figure 3D). The clonal cell line with the desired HDR event underwent an additional round of manual clone picking.

\section{Integrated, Inducible, and Isogenic Lower Motor Neuron (i ${ }^{3} \mathrm{LMN}$ ) Differentiation}

Induced pluripotent stem cells were differentiated into integrated, inducible, and isogenic lower motor neurons ( $\mathrm{i}^{3} \mathrm{LMNs}$ ) as described in Fernandopulle et al. (2018), with the following modifications. On day 3, cells used for immunocytochemistry were seeded at $5-8 \times 10^{4}$ cells per $\mathrm{cm}^{2}$ onto poly-D-lysine (PDL) (Sigma, P7405) and laminin-coated 12-mm glass coverslips in 24-well plates or PDL and laminin-coated clear bottom imaging 96-well plates. Cells used for RNA and protein assays were seeded at $1-1.5 \times 10^{5}$ cells per $\mathrm{cm}^{2}$ onto PDL and laminin-coated 6well plates. On day 4, the media was removed and replaced with fresh Neural Induction Media (NIM) supplemented with B-27 (Gibco, 17504-044), CultureOne (Gibco, A33202-01), $1 \mu \mathrm{g} / \mathrm{mL}$ laminin, $20 \mathrm{ng} / \mathrm{mL}$ BDNF (PeproTech, 450-02), $20 \mathrm{ng} / \mathrm{mL}$ GDNF (PeproTech, 450-10), and $10 \mathrm{ng} / \mathrm{mL}$ NT3 (PeproTech, 450-03). On day 7, a half volume of media was aspirated and replaced with fresh NIM supplemented with B27, CultureOne, $1 \mu \mathrm{g} / \mathrm{mL}$ laminin, $40 \mathrm{ng} / \mathrm{mL}$ BDNF, $40 \mathrm{ng} / \mathrm{mL}$ GDNF, and $20 \mathrm{ng} / \mathrm{mL} \mathrm{NT3}$. On day 10, a half volume of media was aspirated and replaced with fresh Neural Maintenance Media (NMM) supplemented with B-27, CultureOne, $1 \mu \mathrm{g} / \mathrm{mL}$ laminin, $40 \mathrm{ng} / \mathrm{mL}$ BDNF, $40 \mathrm{ng} / \mathrm{mL}$ GDNF, and $20 \mathrm{ng} / \mathrm{mL}$ NT3.

\section{Immunofluorescent Staining}

Integrated, inducible, and isogenic lower motor neurons used for immunofluorescent staining were seeded onto glass coverslips or clear-bottom imaging 96-well plates on day 3 of the differentiation, which then proceeded as previously described. For fixation, an equivalent volume 
of 4\% paraformaldehyde (PFA, Alfa Aesar, J61899AK) was added directly to the cell culture media. Cells were incubated in PFA at room temperature for $20 \mathrm{~min}$, then washed with PBS with $0.1 \%$ Triton-X (Sigma, X100) (PBST) once quickly, followed by two 15 -min washes. The cells were blocked and permeabilized for $1 \mathrm{~h}$ at room temperature with 5\% BSA (Sigma, A4503) in PBS-T. The cells were incubated in primary antibodies at appropriate dilutions (Supplementary Table 3) in blocking buffer at $4^{\circ} \mathrm{C}$ overnight. The following day, the cells were washed with PBS-T at room temperature once quickly, followed by three 10-min washes. The cells were incubated in fluorescent conjugated secondary antibodies diluted at 1:500 in blocking buffer for $1 \mathrm{~h}$ at room temperature. The cells were washed with PBS$\mathrm{T}$ once quickly, followed by three 10 -min washes. The first 10-min wash contained DAPI dye (Invitrogen, D1306). The coverslips were then mounted onto a slide with VECTASHIELD HardSet Mounting Media (Vector Laboratories, H-1400-10). Images were taken on a Keyence BZ-9000 Fluorescence Microscope or an ImageXpress Micro Confocal system (Molecular Devices).

\section{RNA Expression Assays}

Integrated, inducible, and isogenic lower motor neurons used for RNA-based assays were collected on day 7 of the differentiation. RNA was extracted from iPSCs and i ${ }^{3}$ LMNs using the QuickRNA Miniprep Kit (Zymo Research, R1055), according to the manufacturer's instructions. RNA concentrations were quantified using the NanoDrop spectrophotometer. Reverse transcription was performed using the iScript cDNA Synthesis Kit (BioRad, 1708891).

To determine mRNA expression for HB9, choline acetyltransferase (CHAT), and NEFL, we used the respective TaqMan gene expression assays (Thermo Fisher Scientific) containing a FAM-labeled probe. All reactions included a GAPDH gene expression assay (Bio-Rad) containing a HEX-labeled probe, which was used as an internal control to normalize levels of mRNA. $5 \mathrm{ng}$ of cDNA were used in the $25-\mu \mathrm{L}$ reactions measuring $H B 9$ and CHAT expression. $0.25 \mathrm{ng}$ of cDNA were used in the $25-\mu \mathrm{L}$ reactions measuring NEFL expression. The gene expression assay IDs are listed in Supplementary Table 4. To determine allele-specific NEFL mRNA expression, we used a TaqMan SNP genotyping assay targeting the rs79736124 SNP in the $3^{\prime}$ UTR of NEFL (Thermo Fisher Scientific, C_105316276_10). 0.25 ng of cDNA were used in each $25-\mu \mathrm{L}$ ddPCR reaction.

Each $25-\mu \mathrm{L}$ ddPCR reaction contained $12.5 \mu \mathrm{L} 2 \times$ SuperMix for Probes (no dUTP) (Bio-Rad Laboratories, \#186-3024), cDNA, primer and probe mix(es), and water to $25 \mu \mathrm{L}$. Droplets were generated using $20 \mu \mathrm{L}$ of reaction mixture and $70 \mu \mathrm{L}$ of oil with the QX200 Droplet Generator. Droplets were transferred to a 96well PCR plate, sealed, and run on a C1000 Thermal Cycler with a deep well block (Bio-Rad Laboratories). All ddPCR reactions were run under the following thermal cycling conditions: (1) $95^{\circ} \mathrm{C}$ for $10 \mathrm{~min}$; (2) $94^{\circ} \mathrm{C}$ for $30 \mathrm{~s}$; (3) $58^{\circ} \mathrm{C}$ for $1 \mathrm{~min}$; (4) steps 2 and 3 repeat 39 times; (5) $98^{\circ} \mathrm{C}$ for $10 \mathrm{~min}$.
All ddPCR runs were analyzed using the Bio-Rad QuantaSoft Pro Software. The ratio of FAM-positive droplets to HEX positive-droplets was used to calculate the gene expression levels for HB9, CHAT, and NEFL. Fractional abundance of FAM and VIC-positive droplets was used to calculate the percentage of wild-type and mutant NEFL mRNA, respectively.

\section{Western Blots}

Integrated, inducible, and isogenic lower motor neurons were harvested on day 10 of the differentiation and lysed in RIPA buffer (Thermo Fisher Scientific, 89901) with Protease Inhibitor (MedChemExpress, HY-K0010). The samples were incubated for $15 \mathrm{~min}$ on ice and sonicated for $8 \mathrm{~s}$ using a probe sonicator. The samples were then incubated for $10 \mathrm{~min}$ on ice and centrifuged at $10,000 \mathrm{rpm}$ for $10 \mathrm{~min}$ at $4^{\circ} \mathrm{C}$. The supernatant of each sample was transferred to a clean tube. The protein was quantified using the Bio-Rad Protein Assay Dye Reagent Concentrate (BioRad, 5000006).

Samples containing $18 \mu \mathrm{g}$ of total protein were mixed with NuPAGE Sample Reducing Agent (Thermo Fisher Scientific, NP0009) and NuPAGE LDS Sample Buffer (Thermo Fisher Scientific, NP0008) and incubated at $70^{\circ} \mathrm{C}$ for $10 \mathrm{~min}$. The reduced samples were electrophoresed in a $4-12 \%$ Bis-Tris gel. Protein was transferred onto an iBlot nitrocellulose membrane (Thermo Fisher Scientific, IB301002) using the iBlot system (Thermo Fisher Scientific). The membrane was incubated in Odyssey Blocking Buffer (Li-Cor, 927-4000) and PBS at a 1:1 ratio (blocking buffer) for $1 \mathrm{~h}$ at room temperature. Primary antibodies at appropriate dilutions (Supplementary Table 3) were prepared in blocking buffer with $0.1 \%$ Tween 20 (Sigma, 9005-64-5). The membrane was incubated in the primary antibody solution overnight at $4^{\circ} \mathrm{C}$. The following day, the membrane was washed with PBS with $0.1 \%$ Tween 20 (PBS-T) once quickly and with TBS (Corning, 46-012-CM) with 0.1\% Tween 20 (TBS-T) three times for $10 \mathrm{~min}$ each. Fluorescent conjugated secondary antibodies at appropriate dilutions were prepared in blocking buffer. The membrane was incubated in the secondary antibody solution for $1 \mathrm{~h}$ at room temperature. The membrane was then washed with TBS-T 3 times for 10 min each and imaged on the Li-Cor Odyssey Fc Imaging System. Protein band intensity was quantified using ImageJ software. NF-L levels were normalized to GAPDH by dividing NF-L signal intensity by GAPDH signal intensity for each biological replicate.

\section{Image Analysis}

To assess the accumulation of NF-L in the cell bodies of $\mathrm{i}^{3} \mathrm{LMNs}$, the $i^{3}$ LMNs were fixed and stained with anti-HB9 antibody, anti-NF-L antibody, and DAPI. To maximize consistency and reproducibility of our analysis, all neurons for each experiment were fixed and stained simultaneously using a single aliquot and dilution of each antibody. For the day- $7 \mathrm{i}^{3} \mathrm{LMNs}$, ten images were taken per sample on a Keyence BZ-9000 Fluorescence Microscope. For the day- $14 \mathrm{i}^{3} \mathrm{LMNs}$, nine images were taken per sample on an ImageXpress Micro Confocal system (Molecular Devices). All images for each experiment were acquired in a single session with constant illumination and exposure parameters. The cell bodies of $i^{3} \mathrm{LMNs}$ were then segmented manually, or 
automatically using CellProfiler. Before manual segmentation, the images were renamed with alphanumeric codes to allow for blinded analysis. Using ImageJ software, the cell bodies of HB9positive $i^{3}$ LMNs were manually segmented by a blinded observer. Fluorescence intensity was measured in resulting regions of interest from the green channel to measure NF-L intensity within each cell body. To allow for automatic segmentation in later experiments, we designed a CellProfiler pipeline to define cell bodies with $\mathrm{HB} 9$-positive nuclei and measure the average NFL intensity of each cell body (Supplementary Figure 5). For each sample, the average NF-L intensity in the cell bodies was calculated by averaging the intensity values of all the cell bodies across 9-10 images.

\section{NF-L ELISA Assay}

Integrated, inducible, and isogenic lower motor neurons used for the NF-L ELISA Assay were seeded at 30,000 cells per well in a 96-well plate on day 3 of the differentiation. On day 11, a full media change was performed. On day 14, the media from each well was collected and stored at $-80^{\circ} \mathrm{C}$ until the assay was performed. The samples were diluted with water at a 1:25 ratio before quantification. NF-L levels in the media were quantified using the NF-light ELISA kit (Uman Diagnostics, 10-7001).

On day 14 , the cells were imaged using the Incucyte S3 Live-Cell Analysis System. Three images per well were analyzed using CellProfiler software to determine the average neurite area (Supplementary Figure 8). NF-L levels were normalized to the neurite area by dividing the NF-L concentration by the average neurite area for each well.

\section{Multi-Electrode Array Analysis}

Induced pluripotent stem cells were differentiated into $i^{3} \mathrm{LMNs}$ as described in Fernandopulle et al. (2018), with the following modifications. On day 3, cells were seeded at 20,000 cells per well in $10 \mu \mathrm{L}$ of NIM supplemented with $10-\mu \mathrm{M}$ $\mathrm{Y}-27632,2 \mu \mathrm{g} / \mathrm{mL}$ doxycycline, $40-\mathrm{mM}$ BrdU, and 2-mM Compound $\mathrm{E}$ onto polyethylenimine (PEI) (Thermo Fisher Scientific, AC178571000) and laminin-coated 24-well CytoView microelectrode array (MEA) plates (Axion Biosystems, M384tMEA-24W). After the cells were incubated for $20 \mathrm{~min}$ at $37^{\circ} \mathrm{C}$, $500 \mu \mathrm{L}$ of complete media was gently added to each well. On day 4, the media was removed and replaced with fresh NIM supplemented with B-27, CultureOne, $1 \mu \mathrm{g} / \mathrm{mL}$ laminin, $20 \mathrm{ng} / \mathrm{mL}$ BDNF, $20 \mathrm{ng} / \mathrm{mL}$ GDNF, and $10 \mathrm{ng} / \mathrm{mL}$ NT3. On day 7 , a half volume of media was aspirated and replaced with fresh NIM supplemented with B-27, CultureOne, $1 \mu \mathrm{g} / \mathrm{mL}$ laminin, $40 \mathrm{ng} / \mathrm{mL}$ BDNF, $40 \mathrm{ng} / \mathrm{mL}$ GDNF, and $20 \mathrm{ng} / \mathrm{mL}$ NT3. Starting on day 10, every 3 days, a half volume of media was aspirated and replaced with BrainPhys media (StemCell, 05791) supplemented with B-27, CultureOne, $1 \mu \mathrm{g} / \mathrm{mL}$ laminin, $40 \mathrm{ng} / \mathrm{mL}$ BDNF, $40 \mathrm{ng} / \mathrm{mL}$ GDNF, and $20 \mathrm{ng} / \mathrm{mL}$ NT3. Spontaneous neural activity was measured for 15 min using the Axion Biosystem Maestro Edge MEA systems. Data was acquired at $12.5 \mathrm{kHz}$, and spikes were detected in the AxIS Navigator software with an adaptive threshold crossing set to six times the standard deviation of the estimated noise for each electrode. The electrical activity was analyzed using weighted mean firing rate to exclude non-active electrodes for the entire 15-min recording.

\section{RESULTS}

\section{Rapid Development of CMT2E Phenotypes in iPSC-Derived Motor Neurons}

Multiple groups have demonstrated that the NEFL N98S mutation leads to a pathologic accumulation of NF-L in the cell bodies of human and mouse neurons in vitro and in vivo (Adebola et al., 2015; Saporta et al., 2015; Zhao et al., 2017). However, previous methods used to identify phenotypes in motor neurons from CMT2E patient-derived iPSCs were time consuming, often taking $\sim 6$ weeks in total (Saporta et al., 2015). To overcome this challenge, we engineered patient-derived iPSCs with integrated, inducible, and isogenic $\left(i^{3}\right)$ transcription factors in order to rapidly and efficiently differentiate them into lower motor neurons (i $\left.{ }^{3} \mathrm{LMNs}\right)$ (Fernandopulle et al., 2018). We inserted a doxycycline-inducible cassette containing NGN2, ISL1, and LHX3 into the AAVS1 safe-harbor locus (Supplementary Figure 1) of a previously characterized CMT2E iPSC line with the NEFL N98S mutation, referred to here as N98S (Saporta et al., 2015; Maciel et al., 2020; Watry et al., 2020). For comparison, we used healthy control (WT) iPSC lines containing the same doxycycline-inducible cassette integrated into AAVS1 or CLYBL, as previously described (Fernandopulle et al., 2018; Shi et al., 2018). Characterization of the complete transcriptome, chromatin accessibility, and chromatin interactions of our WT $\mathrm{i}^{3} \mathrm{LMNs}$ has been previously published (Song et al., 2019). The publicly available dataset demonstrates that $i^{3} \mathrm{LMNs}$ express a combination of both mature (TUBB3, MAP2, MAPT, SYN1, NEFM, CHAT, and HB9) and immature (NES and DCX) neuronal genes. We confirmed that at day 7 of the differentiation, $i^{3}$ LMNs derived from both WT and N98S lines expressed neuronal markers, including neurofilaments, $\beta$-III tubulin, and the motorneuron transcription factor HB9 (MNX1 gene) (Figure 1A). Consistent with these results, gene expression analysis by reverse transcriptase-droplet digital polymerase chain reaction (RTddPCR) demonstrated that WT and N98S $\mathrm{i}^{3} \mathrm{LMNs}$ expressed MNX1 and the more mature motor neuron marker CHAT at day 7 of the differentiation (Figure 1B). To further verify the functionality of our patient-derived $i^{3} \mathrm{LMNs}$, we performed electrophysiological analysis using a MEA device. We detected rare spontaneous action potentials on day 7 that progressively increased in frequency for both cell lines without a measurable defect in the firing rate of the N98S $\mathrm{i}^{3} \mathrm{LMNs}$ (Figure 1C).

To confirm that we could detect disease-relevant phenotypes at early time points in our differentiation, we fixed WT and N98S $i^{3} \mathrm{LMNs}$ and performed immunofluorescent staining for neurofilament light chain (NF-L) and HB9 using commercially available antibodies (Figures 2A,B). We observed dense accumulation of neurofilament staining in N98S $\mathrm{i}^{3} \mathrm{LMN}$ cell bodies as early as day 7 of differentiation that was not seen in control cells (Figures 1A, 2). For quantification, NF-L 
A
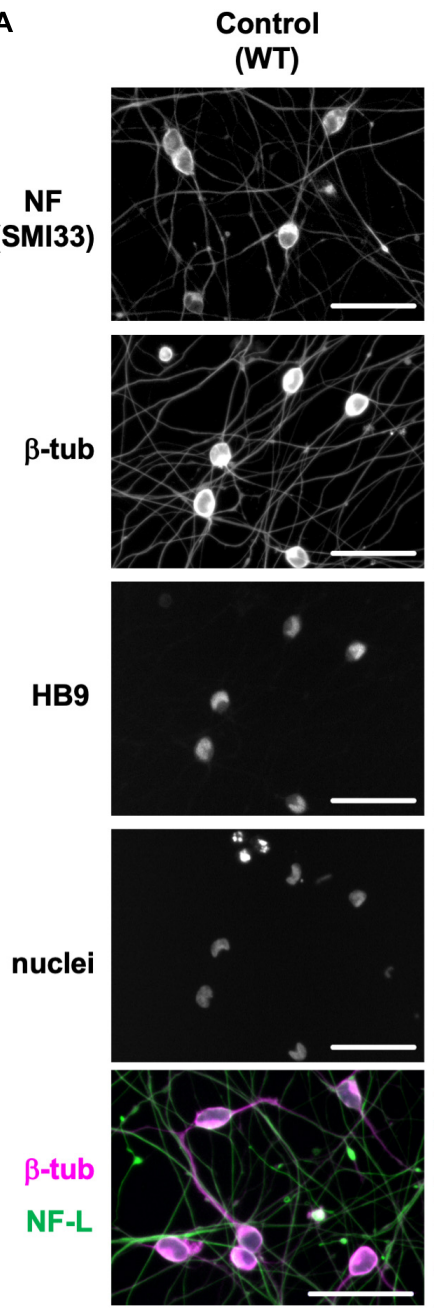

CMT2E

(N98S)
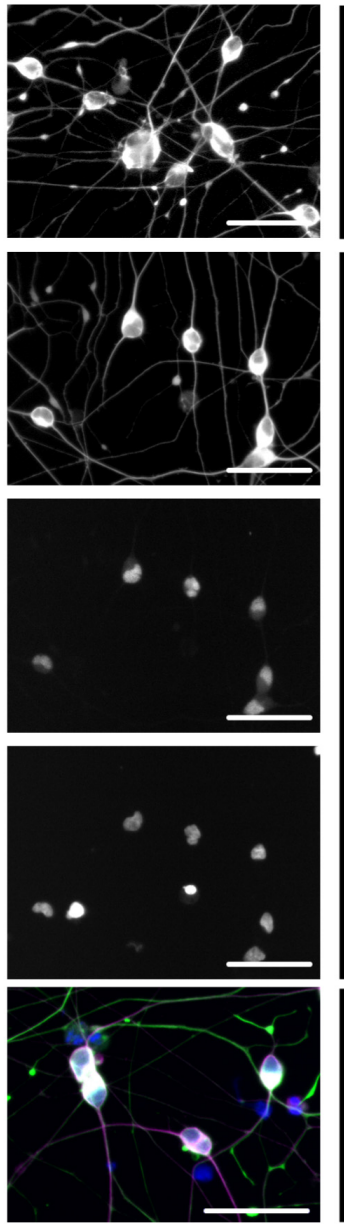

B

\section{Motor Neuron Gene Expression}

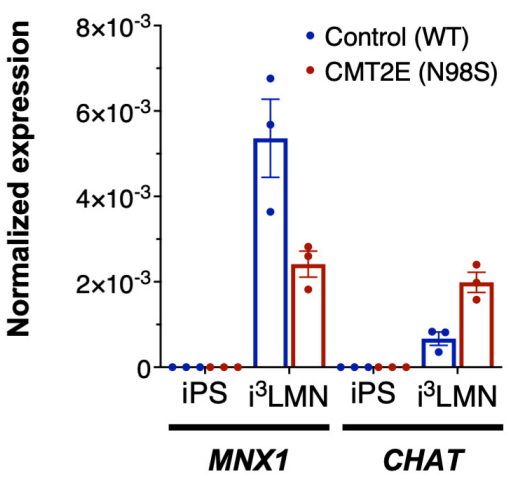

C Spontaneous Electrical Activity

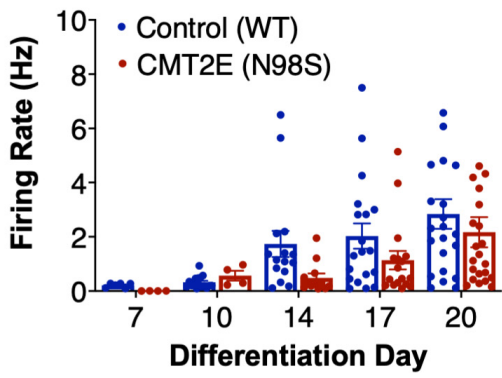

FIGURE 1 | Generation of CMT2E (NEFL-N98S) motor neurons. (A) Expression of neuronal markers by immunofluorescent staining in day-7 i ${ }^{3}$ LMNs derived from healthy control and CMT2E patient iPSC lines. Lower panels are separate RGB channels from the same images, as indicated by a vertical bar. Scale bars $=50 \mu \mathrm{m}$. $\mathrm{NF}=$ neurofilaments (SMI33 antibody), NF-L = neurofilament light chain, $\beta$-tub = $\beta$ III-tubulin, DAPI stain for nuclei. (B) Total mRNA isolated from undifferentiated iPSCs and day- $7 \mathrm{i}^{3}$ LMNs was assayed by RT-ddPCR for expression of motor neuron specific genes MNX1 and CHAT, normalized to GAPDH. (C) WT and N98S $i^{3} L M N$ were seeded on an MEA plate on day 3 of differentiation and spontaneous electrical activity was recorded starting on day 7 and every few days until day 20. Graph represents serial measurements of the mean firing rate (frequency of action potentials) from replicate wells. MEA data were analyzed using a mixed effects model for repeated measurements (maximum likelihood method). There was a significant effect due to time $(p<0.005)$ but no significant difference between cell lines. For all graphs individual replicate data points are shown with bars representing mean \pm S.E.M.

fluorescence intensity in the cell bodies of HB9-positive cell bodies was measured in a blinded fashion. When examining the fluorescence intensity of NF-L staining across all segmented neuronal cell bodies, we observed a marked shift toward highintensity NF-L staining in N98S $\mathrm{i}^{3} \mathrm{LMNs}$, consistent with a misaccumulation phenotype (Figure 2C). To further validate this finding, we compared mean NF-L fluorescence intensities in multiple independent $i^{3} \mathrm{LMN}$ preparations. We observed a greater than 2-fold increase in mean NF-L fluorescence intensity in the cell bodies of N98S $\mathrm{i}^{3} \mathrm{LMNs}$ compared to WT $\mathrm{i}^{3} \mathrm{LMNs}$ (Figure 2D). Thus, we were able to replicate the previously described phenotype, but at a significantly earlier time point compared to other iPSC differentiation methods. We anticipated that this finding would facilitate relatively rapid and sensitive measurement of phenotypic rescue in subsequent gene-editing experiments.

\section{Allele-Specific Editing to Model Therapeutic Strategies in CMT2E iPSCs}

To enable allele-specific silencing of the N98S allele, we designed guide RNAs targeting the N98S sequence (Figure 3A) for two of the most widely used Cas9 enzymes, derived from Streptococcus pyogenes (Sp.) and Staphylococcus aureus (Sa.). We selected a high-fidelity engineered Sp. Cas9 variant (Sp.HiFi) that has been shown to have increased selectivity without compromising activity of the naturally occurring enzyme (Vakulskas et al., 2018). We sought to compare the efficiency and allele specificity of 
A
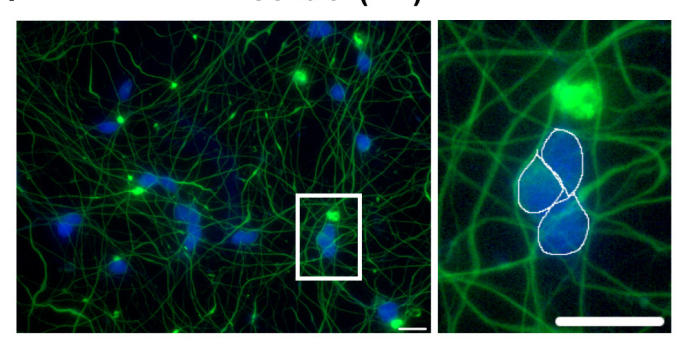

C

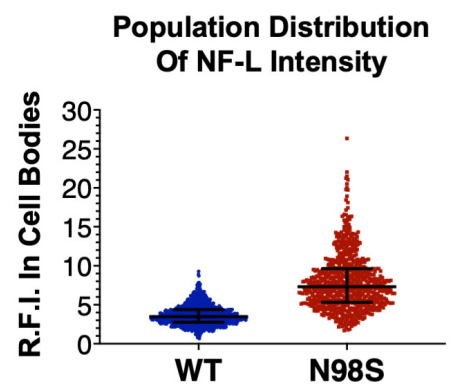

B

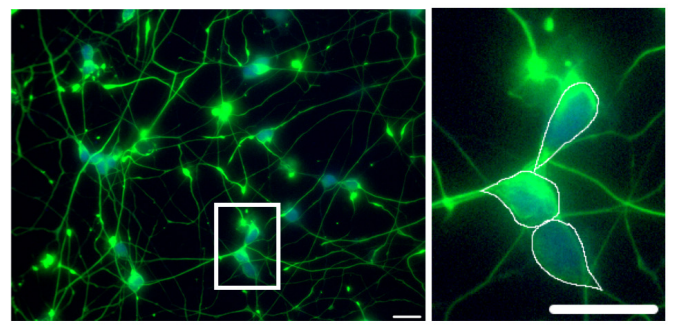

D

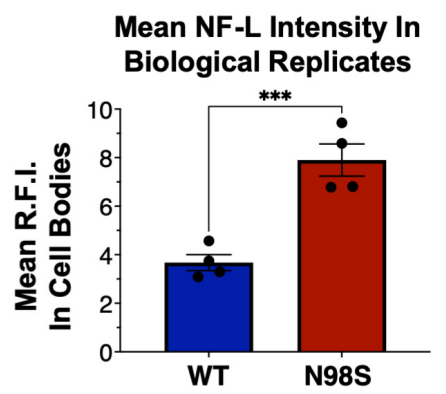

FIGURE 2 | Pathologic accumulation of NF-L protein in the cell body of NEFL-N98S motor neurons. (A,B) Representative images of day-7 WT (A) and N98S (B) ${ }^{3}$ LMNs stained with anti-NF-L (green) and anti-HB9 (blue) antibodies. Boxed regions are shown in larger scale to the right of each image with manual cell body segmentation superimposed. Scale bars $=25 \mu \mathrm{m}$. (C) Distribution of NF-L fluorescence intensity in individual HB9+ cell bodies after manual segmentation. Individual data points are shown with horizontal lines representing median and interquartile range. (D) Quantification of mean NF- $L$ fluorescence intensity from four biological replicates per cell line, demonstrating significantly increased intensity in N98S neurons. Points represent mean of $>160$ neurons quantified in each sample population, bars represent mean of all replicates \pm S.E.M., ${ }^{\star * *} p<0.001$ by $t$-test.

Sp.HiFi Cas9 with Sa. Cas9 as the latter is significantly smaller (315 fewer amino acids), which is advantageous for delivery, in particular via adeno-associated virus vectors. However, the human NEFL sequence lacks a canonical Sa. Cas9 protospacer adjacent motif (PAM) near the N98S mutation so we utilized an engineered variant (Sa.KKH) with an expanded PAM recognition sequence (Kleinstiver et al., 2015). We transfected both N98S and WT iPSCs with the resulting Cas9/gRNA RNP complexes and performed ICE analysis to measure the generation of insertions and deletions (indels) in the target exon. In N98S iPSCs transfected with Sp.HiFi RNP, $45.3 \%$ of NEFL alleles sequenced had indels at the target site (Figure 3B). This is very close to the $50 \%$ maximum expected if all N98S alleles in the heterozygous cell population had been edited. In contrast, in WT iPSCs transfected with Sp.HiFi RNP, we measured 1.7\% of alleles with indels, demonstrating specificity of this nuclease for the N98S allele. In N98S iPSCs transfected with Sa.KKH RNP, we observed moderate rates of editing with $10.5 \%$ of alleles having indels at the target site (Figure 3B). In WT iPSCs transfected with Sa.KKH RNP, we observed no detectable indel generation, demonstrating that this nuclease is also specific for the N98S allele. Among the alleles edited by the Sp.HiFi RNP, we detected two categories of indel events: a 1-bp insertion and a 9-bp deletion (Figure 3C and Supplementary Figure 2). Among the alleles edited by the Sa.KKH RNP, we also detected two categories of indel events, but in this case a 9-bp deletion was predominant, followed by a 15-bp deletion (Figure 3C and Supplementary Figure 2).
The 9- and 15-bp deletion events are unlikely to disrupt expression of the N98S allele as they do not create a shift in reading frame. On the other hand, the 1-bp insertion, which constituted $87.3 \%$ of the total indel events with Sp.HiFi N98S RNP, represents a potential therapeutic edit, as it results in a frameshift mutation. We therefore isolated a clone with a 1-bp insertion at the target site, referred to here as N98Sframeshift (fs). The frameshift mutation leads to a premature stop codon immediately after residue 98 of the N98S allele, which we predicted would effectively interrupt expression from this allele (Supplementary Figure 3B). For comparison, we designed a strategy for precise correction of the N98S allele to a wild-type sequence by HDR. To achieve this, we transfected N98S iPSCs with Sp.HiFi RNP and a single-strand DNA oligonucleotide donor (ssODN). The ssODN was designed to precisely repair the N98S mutation and insert a silent mutation to facilitate genotyping. The efficiency of this editing outcome was low $(2.5 \%)$, so we utilized sib-selection to enrich for cells with the desired HDR event (Miyaoka et al., 2014) (Supplementary Figure 3C). We isolated a clone, referred to as N98S-corrected (N98S-cor), with precise repair of the N98S sequence as well as insertion of silent mutation c.297C > T (Supplementary Figure 3D).

To evaluate for potential off-target editing events produced by the Sp.Hifi RNP, we used the cutting frequency determination (CFD) score to predict the highest risk off-target sequences present in the genome of our cell lines (Doench et al., 2016). We performed targeted amplification and sequencing of 11 genomic 
A

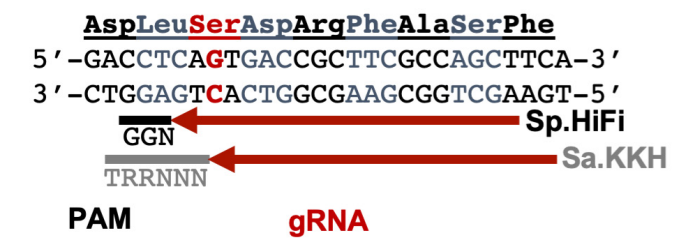

C

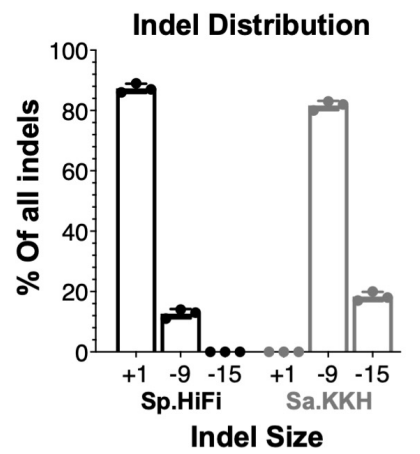

B

Editing Efficiency in iPSC
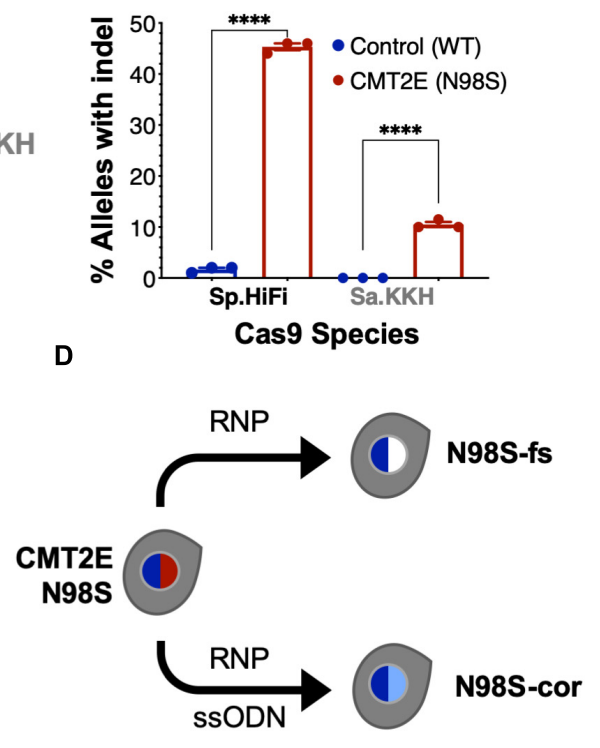

FIGURE 3 | Allele-specific editing of NEFL N98S mutation in iPSCs. (A) NEFL target sequence with N98S mutation in red and gRNAs for Sp.HiFi and Sa.KKH Cas9 targeting the antisense strand. The canonical PAM recognition sequences for Sp.HiFi and Sa.KKH are shown in black and gray, respectively. (B) Efficiency and specificity of indel generation in control and CMT2E patient lines after transfection with Cas9/gRNA ribonucleoprotein (RNP), measured by ICE analysis,

${ }^{* \star \star *} p<0.0001$ by $t$-test. (C) Comparison of indel sizes generated in CMT2E patient line by Cas9 enzyme. (D) Schematic of the derivation of clonal edited iPSC lines from the CMT2E N98S patient line. Transfection of N98S iPSC with Sp.HiFi RNP produced N98S-fs (frameshift inactivating the mutant allele). Transfection of N98S iPSC with Sp.HiFi RNP plus single-strand oligonucleotide donor (ssODN) produced N98S-cor (precise correction of the mutation). Colored nuclei indicate NEFL genotype: dark blue $=$ wild type, red $=$ N98S mutant, white $=$ frameshift (knockout), light blue $=$ correction of N98S to wild type .

loci with CFD score $>0.25$ (Supplementary Table 5) in the parental N98S line and the edited N98S-fs and N98S-cor clones. A heterozygous single-nucleotide deletion (delG) was identified near a predicted target site in an intergenic region of chromosome 13. This deletion was not associated with editing as it was present in all three cell lines and corresponds to a common SNP with allele frequency $>0.4$ in the global population ${ }^{2}$. We did not find evidence of a mutation induced by gene editing in any of the predicted off-target sites.

\section{Frameshift Indel Reduces Mutant NEFL mRNA and Protein Expression in N98S Motor Neurons}

To investigate how allele-specific editing of the N98S allele affects NEFL mRNA and protein expression, we differentiated WT, N98S, N98S-fs, and N98S-cor iPSCs into $\mathrm{i}^{3} \mathrm{LMNs.} \mathrm{In}$ addition, we generated $\mathrm{i}^{3} \mathrm{LMNs}$ from previously characterized iPSC lines with heterozygous $(N E F L+/-)$ and homozygous (NEFL-/-) excision of the NEFL coding exons (Watry et al., 2020). NEFL+/ - and NEFL $-/-\mathrm{i}^{3} \mathrm{LMNs}$ are isogenic to our WT control and were used as a comparison for NEFL mRNA and NF-L protein expression. We measured total NEFL mRNA levels in $i^{3} L M N s$ by quantitative RT-ddPCR and found that N98S-fs $i^{3}$ LMNs had 52\% less NEFL mRNA than their unedited

${ }^{2}$ https://www.ncbi.nlm.nih.gov/snp/rs34866221 counterparts (Figure 4A). This marked reduction in mRNA levels suggested that the early stop codon induced by the heterozygous frameshift mutation may trigger efficient nonsense mediated decay of the mutant transcript. By comparison, NEFL+/ $-\mathrm{i}^{3} \mathrm{LMNs}$ had $36 \%$ less NEFL mRNA than WT $i^{3}$ LMNs (Figure 4A). Thus, the frameshift introduced by the 1-bp insertion disrupted expression of the edited allele as effectively as complete excision of the coding sequence. WT, N98S, and N98S-cor $i^{3}$ LMNs all exhibited similar levels of NEFL mRNA expression. To compare changes in transcription with protein levels, we measured NF-L protein by western blot in $\mathrm{i}^{3} \mathrm{LMNs}$ from the same panel of iPSC lines. Like NEFL mRNA levels, NF-L protein levels were reduced (by 42\%) in N98S-fs $i^{3}$ LMNs compared to N98S $i^{3} L M N s$ (Figure 4B). Interestingly, $N E F L+/-\mathrm{i}^{3} \mathrm{LMNs}$ had a non-significant reduction in NF-L protein compared to WT $\mathrm{i}^{3} \mathrm{LMNs}$. The reason for the differing degrees of NF-L protein reduction in NEFL $+/-$ and N98S-fs $i^{3}$ LMNs is not clear, but may be due to differences in compensatory gene expression, epigenetic effects, or posttranscriptional regulation. WT, N98S, and N98S-cor $\mathrm{i}^{3} \mathrm{LMNs}$ exhibited similar levels of total NF-L protein. Thus, the accumulation of NF-L protein in the cell bodies of N98S $\mathrm{i}^{3} \mathrm{LMN}$ is unlikely to be caused by perturbations in protein synthesis or degradation, but is more consistent with a defect in localization and intracellular transport as has been suggested by other in vitro studies of NF-L mutations (Perez-Olle et al., 2004; Pérez-Ollé et al., 2005). Neither NEFL mRNA nor NF-L 
A

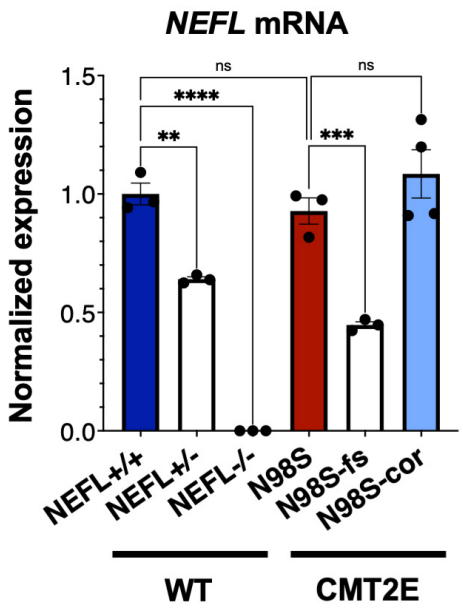

B

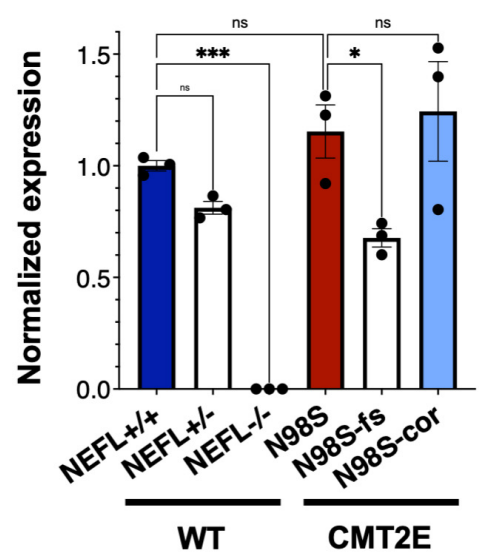

C

Relative Allelic Expression Using Linked SNP
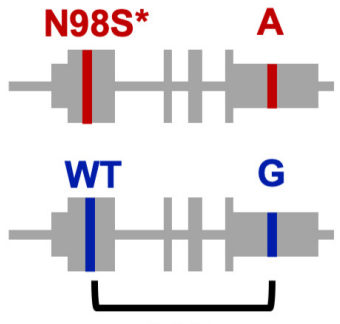

$4.1 \mathrm{~kb}$

RT-ddPCR

probes for SNP A / G

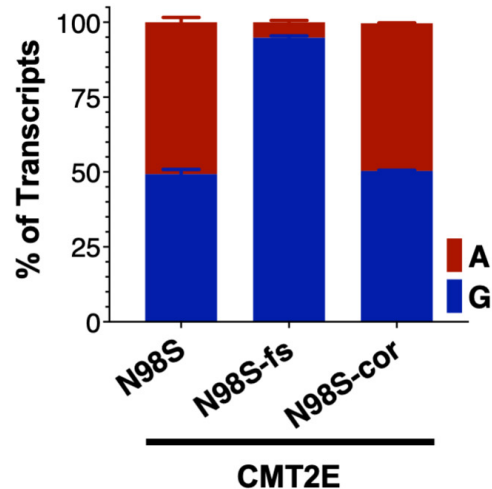

CMT2E

FIGURE 4 | NEFL expression in allelic series of edited motor neurons. A series of edited iPSCs derived from a healthy control (WT) and CMT2E patient were differentiated into i ${ }^{3} L M N s$, and RNA was extracted on day 7 or protein on day 10. (A) Total NEFL transcript expression by quantitative RT-ddPCR, normalized to GAPDH. (B) Total NF-L protein expression by western blot, normalized to GAPDH. (C) Relative expression of $N E F L$ alleles in $\mathrm{i}^{3} \mathrm{LMNs}$ derived from CMT2E patient line and edited subclones. Allele-specific RT-ddPCR targeting a heterozygous SNP in the $3^{\prime}$ UTR was used to genotype transcripts using linkage shown in the schematic. All bar graphs represent mean \pm S.E.M. For all graphs ns $=$ not significant, ${ }^{*} p<0.05,{ }^{* \star} p<0.01,{ }^{* \star *} p<0.001,{ }^{\star \star \star *} p<0.0001$ by one-way ANOVA with Šídák's test for multiple comparisons.

protein was detected in the NEFL-/- samples, validating the specificity of our assays.

To confirm that the observed decrease in total NEFL expression in N98S-fs $i^{3} \mathrm{LMNs}$ was specific to the mutant allele, we measured the relative expression of the mutant and wild-type alleles in N98S, N98S-fs, and N98S-cor $\mathrm{i}^{3}$ LMNs. Allele specificity was achieved by targeting a RT-ddPCR assay to a heterozygous SNP present in the $3^{\prime}$ untranslated region of the NEFL gene in our CMT2E patient line (Figure 4C). In N98S and N98S-cor $i^{3} \mathrm{LMNs}$, both alleles were transcribed at equal rates, with each representing $50 \%$ of the total transcripts detected. However, in N98S-fs $i^{3}$ LMNs, NEFL mutant allele transcripts constituted only $5 \%$ of the total NEFL transcripts detected, confirming efficient knockdown of the mutant mRNA (Figure 4C). Overall, these experiments demonstrate that the frameshift mutation leads to dramatic reduction of mutant NEFL mRNA and, presumably, mutant NF-L protein in $i^{3} \mathrm{LMNs}$, while isogenic correction of the
N98S mutation with introduction of a silent mutation does not alter NEFL expression.

\section{Selective Inactivation of the N98S Allele Rescues Pathologic Phenotypes in Motor Neurons}

To determine if inactivation of the NEFL mutant allele prevents the pathologic phenotype, we measured the distribution of NF-L in fixed and stained WT, N98S, N98S-fs, and N98S-cor i ${ }^{3}$ LMNs at day 7 (Figures 5A-C). To increase the throughput of this analysis, we developed an automated image analysis pipeline using CellProfiler to identify $\mathrm{i}^{3} \mathrm{LMN}$ cell bodies and quantify NF-L fluorescence intensity (Figure 5C and Supplementary Figure 5). Consistent with the manually processed data, N98S $\mathrm{i}^{3} \mathrm{LMN}$ cell bodies displayed significantly higher NF-L fluorescence intensity values compared to WT. N98S-fs $\mathrm{i}^{3} \mathrm{LMNs}$ displayed 
A

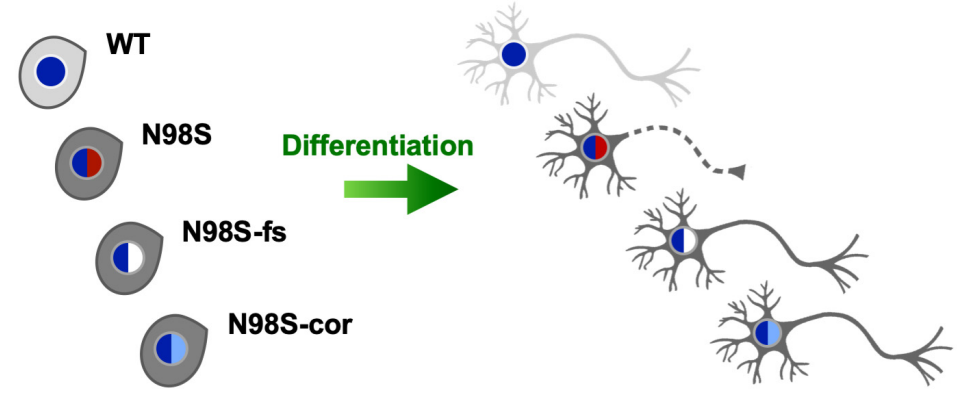

B
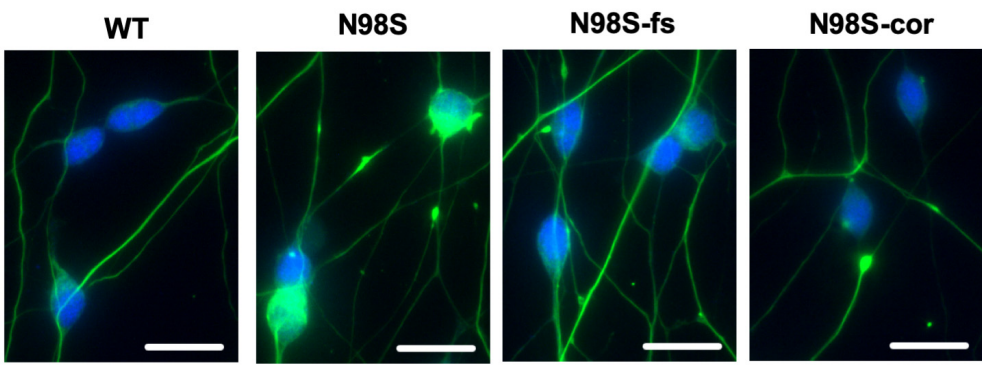

C

Automated Image Analysis

D
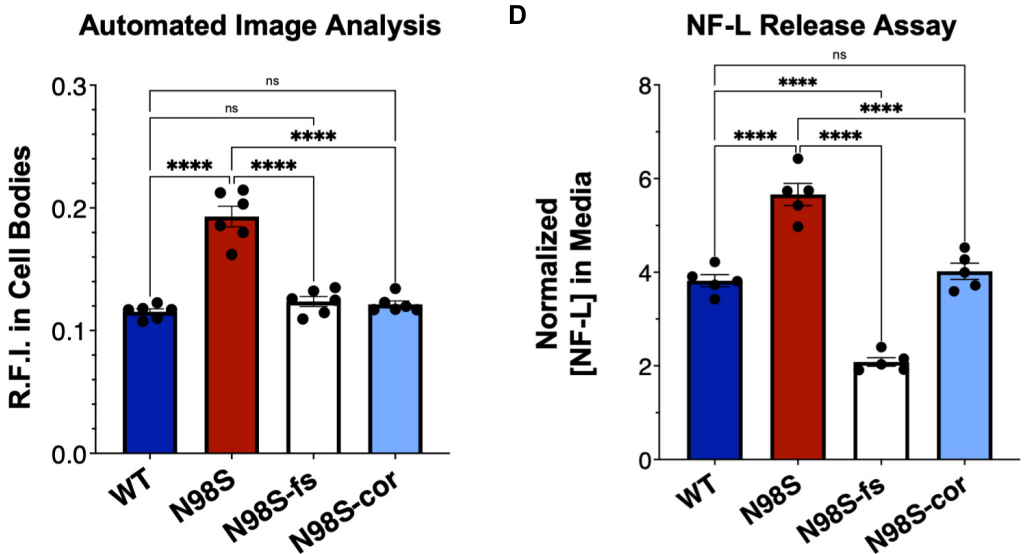

FIGURE 5 | Rescue of pathologic phenotypes in edited N98S motor neurons. (A) Schematic of allelic series of iPSCs differentiated into healthy (continuous axon) or diseased (dashed axon) i ${ }^{3}$ LMNs. Colored nuclei represent NEFL genotypes as described in Figure 3. Cytoplasmic shading indicates differential genetic backgrounds, dark gray cells are isogenic. (B) Representative images of day- $7 \mathrm{i}^{3} \mathrm{LMNs}$ stained with anti-NF-L (green) and anti-HB9 (blue) antibodies. Scale bars $=25 \mu \mathrm{m}$. (C) Quantification of NF-L relative fluorescence intensity (R.F.I.) in HB9+ cell bodies using automated image analysis pipeline. Data points represent mean values from independent differentiations, bars indicate mean of six biological replicates \pm S.E.M. (D) ELISA for NF-L protein in media from day-14 $i^{3} L M N s$. NF-L levels were normalized to neurite density measurements to correct for well-to-well variability in cell seeding (Supplementary Figure 8). Individual data points shown, bars indicate mean of five biological replicates \pm S.E.M. For all graphs ${ }^{\star \star \star \star} p<0.0001$ by one-way ANOVA with Šídák's test for multiple comparisons.

significantly lower NF-L fluorescence intensity values than N98S $i^{3}$ LMNs, demonstrating that inactivation of the mutant NEFL allele prevents the pathologic accumulation of NF-L in the cell bodies of motor neurons. In addition, the NF-L fluorescence intensity values of N98S-fs $\mathrm{i}^{3} \mathrm{LMNs}$ were indistinguishable from those of WT and N98S-cor i ${ }^{3}$ LMNs, further suggesting that NF$\mathrm{L}$ localization and transport is normalized after inactivation of the N98S mutant allele. To determine whether rescue of this phenotype would persist to later points in differentiation, we repeated the analysis at day 14 (Supplementary Figure 6). Again, N98S-fs and N98S-cor $\mathrm{i}^{3} \mathrm{LMNs}$ had significantly decreased NF$\mathrm{L}$ fluorescence intensity in the cell bodies compared to N98S $\mathrm{i}^{3} \mathrm{LMNs}$, to a level indistinguishable from WT $\mathrm{i}^{3} \mathrm{LMNs}$. We did observe a smaller magnitude of difference between N98S and the other cell lines at day 14 in this assay, likely because our analysis pipeline was optimized for the earlier time point when neuron cell bodies are less tightly clustered. We also investigated whether gene editing altered the ability of the neurons to generate functional action potentials. MEA analysis demonstrated that the N98S-fs and N98S-cor $\mathrm{i}^{3} \mathrm{LMNs}$ retained similar spontaneous electrical activity as their unedited N98S counterparts (Supplementary Figure 7).

Finally, we assayed NF-L release in the culture media as a means to assess cellular integrity of our $\mathrm{i}^{3} \mathrm{LMNs}$. NF-L is 
a biomarker of neurodegenerative diseases, as NF-L levels in cerebrospinal fluid and serum reflect the extent of neuroaxonal damage leading to neurofilament release (Lu et al., 2015; Disanto et al., 2017; Hansson et al., 2017). Correspondingly, it has been reported that iPSC-derived motor neuron cultures with the NEFL-N98S mutation release more NF-L into the media than healthy controls, consistent with increased axonal degeneration (Maciel et al., 2020). Using a commercially available enzymelinked immunosorbent assay (ELISA), we measured the levels of NF-L in media from day-14 $\mathrm{i}^{3} \mathrm{LMN}$ cultures (Figure 5D). As expected, media from N98S $i^{3}$ LMNs had significantly higher normalized levels of NF-L than media from WT $\mathrm{i}^{3} \mathrm{LMNs}$, suggesting that the N98S mutation results in neuroaxonal damage. N98S-cor and WT ${ }^{3}$ LMN cultures exhibited equivalent levels of NF-L, confirming that the increase in NF-L levels observed in N98S $\mathrm{i}^{3} \mathrm{LMNs}$ was due to the N98S mutation rather than inherent cell line variability. In contrast, N98S-fs $i^{3}$ LMNs released significantly less NF-L than all other cell lines, including the WT and N98S-cor controls. This was expected, as overall NF-L expression is reduced in N98S-fs $\mathrm{i}^{3} \mathrm{LMNs}$ (Figure 4B). To determine whether this observation was simply due to changes in intracellular protein expression, we compared cell lines by differences in NF-L released into media (as measured by ELISA, Figure 5D) versus differences in intracellular NF-L levels (as measured by western blot, Figure 4B). The relative ratios of N98S-fs to N98S-cor were similar: 0.52 for NF-L released in media and 0.55 for intracellular NF-L. This demonstrates that the differences in released NF-L can be completely explained by intracellular protein levels and suggests equivalent degrees of neuronal integrity when comparing the two editing strategies. Conversely, the relative ratios of N98S-fs to parental N98S were 0.37 for NF-L released in media and 0.59 for intracellular NFL. The larger decrease in NF-L released into the media supports the conclusion that inactivation of the mutant allele leads to both decreased NF-L protein expression, as well as improved neuronal integrity compared to the unedited diseased neurons.

In summary, we conclude that inactivation of the mutant allele by CRISPR-Cas9 gene editing effectively prevents disease phenotypes in $\mathrm{i}^{3} \mathrm{LMNs}$ derived from a patient with autosomal dominant CMT2E caused by the N98S mutation. Moreover, N98S-fs $i^{3}$ LMNs were phenotypically similar to N98S-cor $i^{3} \mathrm{LMNs}$, suggesting that knockout of the mutant allele is as effective as precise repair of the mutation in this model system.

\section{DISCUSSION}

In this study, we used allele-specific gene editing with engineered Cas9 enzymes to inactivate a missense mutation causing CMT2E, a severe autosomal dominant neuromuscular disease. Cas9induced inactivation was efficient, selective, and effectively reversed the accompanying pathology in patient iPSC-derived motor neurons without apparent adverse effects. In the process, we incorporated rapid neuronal differentiation and highthroughput automated image analysis to enable measurement of changes in disease phenotypes in a fraction of the time required by previous methodology. Our findings establish the framework for therapeutic development of gene editing for CMT2E and for other motor neuron diseases caused by dominant missense mutations.

\section{Gene Editing as an Efficient Path to Allele-Specific Gene Inactivation}

We selected CRISPR-Cas9-based genome editing because it presents several advantages over other therapeutic strategies, with important clinical implications (Porteus, 2019). First, the therapeutic construct only needs to be active briefly and requires only a single treatment, since the outcome is a permanent change in the cellular genome. In fact, transient expression is desirable as it minimizes the risk of off-target effects and immune response to the vector construct. Second, since it targets the mutation itself, gene editing can achieve complete correction or inactivation of the mutant allele in treated cells, rather than mere attenuation of the mutation's effect. Finally, even singlenucleotide mutations can be targeted by Cas nucleases with a high degree of specificity (Wienert et al., 2019), suggesting the approach could be safely deployed to treat a large number of conditions caused by missense mutations.

We demonstrated that Cas9 enzymes derived from both $S$. aureus and $S$. pyogenes can induce indels specifically at the NEFL-N98S mutant allele, and that the latter predominantly leads to a 1-bp insertion that efficiently disrupts mutant allele expression. In contrast, the former preferentially generates 9and 15-bp deletions that are less useful for disrupting expression of the mutant allele. Interestingly, 9- and 15-bp deletions are predicted by microhomology domains near the cleavage sites for both of our Cas9 nucleases (Bae et al., 2014). Large-scale studies have begun to define the mutational profiles produced by Cas9induced cleavage and predict outcomes produced by specific gRNA, but our findings highlight how these predictions are not necessarily transferable to alternate enzymes, which differ in the nature of the double-strand breaks produced (Allen et al., 2018; Tycko et al., 2018; Chen et al., 2019). Moreover, these predictions are based on experiments performed in immortalized cell lines and the generalizability to primary and post-mitotic cells is yet to be determined.

We achieved phenotypic rescue using CRISPR gene editing both by generating indels in the NEFL allele carrying the N98S mutation and by correcting the N98S mutation to wild type via $\mathrm{HDR}$. In both cases, gene editing starts with the creation of targeted double-strand breaks, but the outcome depends on the endogenous DNA repair machinery that repairs the breaknon-homologous end-joining (NHEJ) or HDR (Porteus, 2019). NHEJ is most useful for gene inactivation via frameshift-inducing indels, as in our approach, and is active even in post-mitotic neurons (Bétermier et al., 2014; Shalem et al., 2014; Gaj et al., 2017). Precise correction of a mutant sequence by HDR requires a donor template to facilitate homologous recombination but is typically much less efficient than NHEJ and active only in proliferative cells (Miyaoka et al., 2016). Indeed, in our experiments, editing outcomes from NHEJ were over 10 times more efficient than HDR using the same gRNA and Cas9 enzyme. Both approaches were able to effectively rescue disease 
phenotypes in CMT2E $\mathrm{i}^{3} \mathrm{LMNs}$, but as HDR is not active in postmitotic cells like neurons, inactivation of the mutant allele via NHEJ is the more tractable therapeutic strategy. An important caveat is that allele-specific gene inactivation is restricted to genes in which heterozygous loss of function is well tolerated, as is the case for NEFL.

\section{Alternative Strategies and Future Directions}

We validated CRISPR reagents that are specific to the human NEFL-N98S mutation, but there are a multitude of distinct missense mutations in NEFL that cause CMT2E (Stone et al., 2019). The majority occur in the first exon and should be amenable to the same editing strategy that we employed here. However, inducing frameshifts at the site of mutations in distal coding exons may not be therapeutic, as nonsense codons in that context can lead to expression of a pathologic truncated protein rather than nonsense-mediated decay (Lindeboom et al., 2016; Stone et al., 2019). Alternate editing strategies for these mutations include excision of mutant alleles using pairs of guide RNAs, or precise correction using non-nuclease editing technologies such as base editors or PRIME editing (Komor et al., 2016; Anzalone et al., 2019; Watry et al., 2020).

Alternative gene therapy approaches include gene augmentation, more commonly used to treat recessive lossof-function mutations, and RNA interference; however, both present their own challenges. Gene augmentation in dominant disease can have partial benefits (Mao et al., 2011) yet it requires high levels of exogenous gene expression to overcome effects of the mutant allele, and will be ineffective if the mutant gene product continues to interfere with the function of the wild-type gene product, or causes direct toxicity to the cell (Yu-Wai-Man, 2016; Diakatou et al., 2019). RNA interference, where a vector delivers a small RNA that targets the mutant transcript for degradation, has been used successfully in animal models of dominant disease, including CMT2D (Matsa et al., 2014; Morelli et al., 2019; Zaleta-Rivera et al., 2019). Although promising, this approach needs to overcome significant challenges including efficiency and specificity, particularly for single-nucleotide point mutations. Furthermore, both gene augmentation and RNA interference require long-term persistence of the vector or repeated treatments.

We performed our studies in a human motor neuron model system because motor symptoms are typically the most debilitating in CMT2E and many subtypes of axonal CMT, although sensory neurons are also affected (Adebola et al., 2015; Yang et al., 2016; Zhao et al., 2017; Lancaster et al., 2018). Although we focused on motor neurons, a similar approach could be applied to sensory neurons or glial cells differentiated from patient iPSCs. Limitations of our system include the relatively immature developmental state of the motor neurons, absence of other cell types, and lack of anatomic structures. As a result, we are not able to recapitulate all disease phenotypes, such as axonal atrophy, decreased nerve conduction velocity, and length-dependent axonal degeneration, such as that are present in human patients and animal models. Advances in the development of three-dimensional and heterotypic microtissues derived from iPSC have the potential to overcome some of these limitations in the future.

A limitation of our studies is that we performed editing in stem cells rather than in the neurons directly. Efficient delivery of gene-editing reagents to neurons in vitro is challenging, and ongoing work in our group and others is focused on improving methodology to enable routine gene-editing experiments in iPSC-derived neurons. The studies presented here establish a crucial baseline, by identifying candidate therapeutic reagents and validating rapid development of disease-specific phenotypes that can be assayed in high throughput. These tools will be invaluable to answer outstanding questions including whether phenotypic rescue will be variable in patient lines with different genetic backgrounds, applicability to other missense mutations in NEFL, allele specificity and repair outcomes in neurons, and the time course for reversing the established disease phenotype. Furthermore, in vivo animal experiments will be important to complement the in vitro human models. Fortunately, in the case of CMT2E there is an excellent animal model in the N98S knock-in mouse that precisely mimics the human mutation, and is associated with a severe and well characterized phenotype (Adebola et al., 2015; Lancaster et al., 2018). In the case of gene editing, the ultimate therapeutic reagent must be primarily developed and validated in a human system due to speciesspecific genome sequences, but in vivo experiments will be critical to optimize delivery methods, assess functional outcomes, and evaluate systemic side effects.

\section{DATA AVAILABILITY STATEMENT}

The raw data supporting the conclusions of this article will be made available by the authors, without undue reservation.

\section{ETHICS STATEMENT}

The studies involving human participants were reviewed and approved by University of California, San Francisco Human Research Protection Program Institutional Review Board. The patients/participants provided their written informed consent to participate in this study.

\section{AUTHOR CONTRIBUTIONS}

LJ, MS, and BC conceived the project. CF, KW, HW, and LJ designed the experiments. CF performed the majority of experiments and data analysis with assistance from other authors as specified. $\mathrm{KW}, \mathrm{HG}, \mathrm{AL}$, and $\mathrm{HW}$ transfected $\mathrm{PPSC}$ and isolated clonal lines. KW optimized neuron differentiation, western blots, and immunofluorescent staining. CM performed selected gene editing and immunofluorescent staining. CM, LZ, and TM conducted multielectrode array analysis. GR helped optimize neuron differentiation and design of the CellProfiler analysis pipeline. $\mathrm{CF}$ and $\mathrm{LJ}$ wrote the manuscript with assistance from 
all authors. All authors contributed to the article and approved the submitted version.

\section{FUNDING}

LJ and BC received funding from the Charcot-Marie-Tooth Association (CMTA). LJ, BC, and TM received support from NIH grant U01-ES032673. GR received support from the NSF Graduate Research Fellowship Program and the UCSF Discovery Fellowship. LZ received support from the Lisa Dean Moseley Foundation. BC received support from the Gladstone Institutes, Innovative Genomics Institute, and NIH grants R01-EY028249, R01-HL130533, R01-HL135358, P01-HL146366, RF1-AG072052 and the Claire Giannini Fund.

\section{REFERENCES}

Abe, A., Numakura, C., Saito, K., Koide, H., Oka, N., Honma, A., et al. (2009). Neurofilament light chain polypeptide gene mutations in Charcot-Marie-Tooth disease: nonsense mutation probably causes a recessive phenotype. J. Hum. Genet. 54, 94-97. doi: 10.1038/jhg.2008.13

Adebola, A. A., Di Castri, T., He, C.-Z., Salvatierra, L. A., Zhao, J., Brown, K., et al. (2015). Neurofilament light polypeptide gene N98S mutation in mice leads to neurofilament network abnormalities and a Charcot-Marie-Tooth Type 2E phenotype. Hum. Mol. Genet. 24, 2163-2174. doi: 10.1093/hmg/ ddu736

Allen, F., Crepaldi, L., Alsinet, C., Strong, A. J., Kleshchevnikov, V., De Angeli, P., et al. (2018). Predicting the mutations generated by repair of Cas9induced double-strand breaks. Nat. Biotechnol. 37, 64-72. doi: 10.1038/nbt. 4317

Anzalone, A. V., Randolph, P. B., Davis, J. R., Sousa, A. A., Koblan, L. W., Levy, J. M., et al. (2019). Search-and-replace genome editing without double-strand breaks or donor DNA. Nature 576, 149-157. doi: 10.1038/s41586-019-1711-4

Bae, S., Kweon, J., Kim, H. S., and Kim, J.-S. (2014). Microhomology-based choice of Cas9 nuclease target sites. Nat. Methods 11, 705-706. doi: 10.1038/nmeth. 3015

Bétermier, M., Bertrand, P., and Lopez, B. S. (2014). Is non-homologous endjoining really an inherently error-prone process? PLoS Genet. 10:e1004086. doi: 10.1371/journal.pgen.1004086

Brennan, K. M., Bai, Y., and Shy, M. E. (2015). Demyelinating CMT-what's known, what's new and what's in store? Neurosci. Lett. 596, 14-26. doi: 10.1016/j.neulet. 2015.01.059

Cerbini, T., Funahashi, R., Luo, Y., Liu, C., Park, K., Rao, M., et al. (2015). Transcription activator-like effector nuclease (TALEN)-mediated CLYBL targeting enables enhanced transgene expression and one-step generation of dual reporter human induced pluripotent stem cell (iPSC) and neural stem cell (NSC) lines. PLoS One 10:e0116032. doi: 10.1371/journal.pone.0116032

Chen, W., McKenna, A., Schreiber, J., Haeussler, M., Yin, Y., Agarwal, V., et al. (2019). Massively parallel profiling and predictive modeling of the outcomes of CRISPR/Cas9-mediated double-strand break repair. Nucleic Acids Res. 47, 7989-8003. doi: 10.1093/nar/gkz487

Diakatou, M., Manes, G., Bocquet, B., Meunier, I., and Kalatzis, V. (2019). Genome editing as a treatment for the most prevalent causative genes of autosomal dominant retinitis pigmentosa. Int. J. Mol. Sci. 20:2542. doi: 10.3390/ ijms 20102542

Disanto, G., Barro, C., Benkert, P., Naegelin, Y., Schädelin, S., Giardiello, A., et al. (2017). Serum Neurofilament light: a biomarker of neuronal damage in multiple sclerosis. Ann. Neurol. 81, 857-870. doi: 10.1002/ana.24954

DiVincenzo, C., Elzinga, C. D., Medeiros, A. C., Karbassi, I., Jones, J. R., Evans, M. C., et al. (2014). The allelic spectrum of Charcot-Marie-Tooth disease in over 17,000 individuals with neuropathy. Mol. Genet. Genomic Med. 2, 522-529. doi: $10.1002 / \mathrm{mgg} 3.106$

\section{ACKNOWLEDGMENTS}

We thank Michael Ward for providing the AAVS1-hNIL iPSC line and AAVS1-hNIL and CLYBL-hNIL plasmids, the Gladstone Stem Cell Core and Cell Line Genetics for their services, and A. Birk, A. Sachdev, P. Dua, Z. Nevin, C. Clelland, and F. Chanut for technical assistance, advice, and critical review of the manuscript.

\section{SUPPLEMENTARY MATERIAL}

The Supplementary Material for this article can be found online at: https://www.frontiersin.org/articles/10.3389/fcell.2021. 723023/full\#supplementary-material

Doench, J. G., Fusi, N., Sullender, M., Hegde, M., Vaimberg, E. W., Donovan, K. F., et al. (2016). Optimized sgRNA design to maximize activity and minimize offtarget effects of CRISPR-Cas9. Nat. Biotechnol. 34, 184-191. doi: 10.1038/nbt. 3437

Fernandopulle, M. S., Prestil, R., Grunseich, C., Wang, C., Gan, L., and Ward, M. E. (2018). Transcription factor-mediated differentiation of human iPSCs into neurons. Curr. Protoc. Cell Biol. 79:e51. doi: 10.1002/cpcb.51

Fu, J., and Yuan, Y. (2018). A novel homozygous nonsense mutation in NEFL causes autosomal recessive Charcot-Marie-Tooth disease. Neuromuscul. Disord. 28, 44-47. doi: 10.1016/j.nmd.2017.09.018

Gaj, T., Ojala, D. S., Ekman, F. K., Byrne, L. C., Limsirichai, P., and Schaffer, D. V. (2017). In vivo genome editing improves motor function and extends survival in a mouse model of ALS. Sci. Adv. 3, eaar3952. doi: 10.1126/sciadv.aar3952

Hansson, O., Janelidze, S., Hall, S., Magdalinou, N., Lees, A. J., Andreasson, U., et al. (2017). Blood-based NfL: a biomarker for differential diagnosis of parkinsonian disorder. Neurology 88, 930-937. doi: 10.1212/WNL.00000000000 03680

Jordanova, A., De Jonghe, P., Boerkoel, C. F., Takashima, H., De Vriendt, E., Ceuterick, C., et al. (2003). Mutations in the neurofilament light chain gene (NEFL) cause early onset severe Charcot-Marie-Tooth disease. Brain 126, 590-597. doi: 10.1093/brain/awg059

Kleinstiver, B. P., Prew, M. S., Tsai, S. Q., Nguyen, N. T., Topkar, V. V., Zheng, Z., et al. (2015). Broadening the targeting range of Staphylococcus aureus CRISPR-Cas9 by modifying PAM recognition. Nat. Biotechnol. 33, 1293-1298. doi: 10.1038/nbt.3404

Komor, A. C., Kim, Y. B., Packer, M. S., Zuris, J. A., and Liu, D. R. (2016). Programmable editing of a target base in genomic DNA without doublestranded DNA cleavage. Nature 533, 420-424. doi: 10.1038/nature17946

Kreitzer, F. R., Salomonis, N., Sheehan, A., Huang, M., Park, J. S., Spindler, M. J., et al. (2013). A robust method to derive functional neural crest cells from human pluripotent stem cells. Am. J. Stem Cells 2, 119-131.

Lancaster, E., Li, J., Hanania, T., Liem, R., Scheideler, M. A., and Scherer, S. S. (2018). Myelinated axons fail to develop properly in a genetically authentic mouse model of Charcot-Marie-Tooth disease type 2E. Exp. Neurol. 308, 13-25. doi: 10.1016/j.expneurol.2018.06.010

Lindeboom, R. G. H., Supek, F., and Lehner, B. (2016). The rules and impact of nonsense-mediated mRNA decay in human cancers. Nat. Genet. 48, 1112-1118. doi: $10.1038 /$ ng.3664

Lu, C.-H., Macdonald-Wallis, C., Gray, E., Pearce, N., Petzold, A., Norgren, N., et al. (2015). Neurofilament light chain: a prognostic biomarker in amyotrophic lateral sclerosis. Neurology 84, 2247-2257. doi: 10.1212/WNL. 0000000000001642

Luigetti, M., Fabrizi, G. M., Bisogni, G., Romano, A., Taioli, F., Ferrarini, M., et al. (2016). Charcot-Marie-Tooth type 2 and distal hereditary motor neuropathy: clinical, neurophysiological and genetic findings from a singlecentre experience. Clin. Neurol. Neurosurg. 144, 67-71. doi: 10.1016/j.clineuro. 2016.03.007 
Maciel, R., Correa, R., Bosso Taniguchi, J., Prufer Araujo, I., and Saporta, M. A. (2020). Human tridimensional neuronal cultures for phenotypic drug screening in inherited peripheral neuropathies. Clin. Pharmacol. Ther. 107, 1231-1239. doi: $10.1002 /$ cpt.1718

Mao, H., James, T., Schwein, A., Shabashvili, A. E., Hauswirth, W. W., Gorbatyuk, M. S., et al. (2011). AAV delivery of wild-type rhodopsin preserves retinal function in a mouse model of autosomal dominant retinitis pigmentosa. Hum. Gene Ther. 22, 567-575. doi: 10.1089/hum.2010.140

Matsa, E., Dixon, J. E., Medway, C., Georgiou, O., Patel, M. J., Morgan, K., et al. (2014). Allele-specific RNA interference rescues the long-QT syndrome phenotype in human-induced pluripotency stem cell cardiomyocytes. Eur. Heart J. 35, 1078-1087. doi: 10.1093/eurheartj/eht067

Mendell, J. R., Al-Zaidy, S., Shell, R., Arnold, W. D., Rodino-Klapac, L. R., Prior, T. W., et al. (2017). Single-Dose Gene-Replacement Therapy for Spinal Muscular Atrophy. N. Engl. J. Med. 377, 1713-1722. doi: 10.1056/ NEJMoa1706198

Miyaoka, Y., Berman, J. R., Cooper, S. B., Mayerl, S. J., Chan, A. H., Zhang, B., et al. (2016). Systematic quantification of HDR and NHEJ reveals effects of locus, nuclease, and cell type on genome-editing. Sci. Rep. 6:23549. doi: 10.1038/ srep23549

Miyaoka, Y., Chan, A. H., Judge, L. M., Yoo, J., Huang, M., Nguyen, T. D., et al. (2014). Isolation of single-base genome-edited human iPS cells without antibiotic selection. Nat. Methods 11, 291-293. doi: 10.1038/nmeth.2840

Morelli, K. H., Griffin, L. B., Pyne, N. K., Wallace, L. M., Fowler, A. M., Oprescu, S. N., et al. (2019). Allele-specific RNA interference prevents neuropathy in Charcot-Marie-Tooth disease type 2D mouse models. J. Clin. Invest. 129, 5568-5583. doi: 10.1172/JCI130600

Pareyson, D., Saveri, P., and Pisciotta, C. (2017). New developments in CharcotMarie-Tooth neuropathy and related diseases. Curr. Opin. Neurol. 30, 471-480. doi: 10.1097/WCO.0000000000000474

Perez-Olle, R., Jones, S. T., and Liem, R. K. H. (2004). Phenotypic analysis of neurofilament light gene mutations linked to Charcot-Marie-Tooth disease in cell culture models. Hum. Mol. Genet. 13, 2207-2220. doi: 10.1093/hmg/ ddh 236

Pérez-Ollé, R., López-Toledano, M. A., Goryunov, D., Cabrera-Poch, N., Stefanis, L., Brown, K., et al. (2005). Mutations in the neurofilament light gene linked to Charcot-Marie-Tooth disease cause defects in transport. J. Neurochem. 93, 861-874. doi: 10.1111/j.1471-4159.2005.03095.x

Porteus, M. H. (2019). A New Class of Medicines through DNA Editing. N. Engl. J. Med. 380, 947-959. doi: 10.1056/NEJMra1800729

Reilly, M. M., Murphy, S. M., and Laurá, M. (2011). Charcot-Marie-Tooth disease. J Peripher. Nerv. Syst. 16, 1-14. doi: 10.1111/j.1529-8027.2011.00324.x

Rossor, A. M., Evans, M. R. B., and Reilly, M. M. (2015). A practical approach to the genetic neuropathies. Pract. Neurol. 15, 187-198. doi: 10.1136/practneurol2015-001095

Saporta, M. A., Dang, V., Volfson, D., Zou, B., Xie, X. S., Adebola, A., et al. (2015). Axonal Charcot-Marie-Tooth disease patient-derived motor neurons demonstrate disease-specific phenotypes including abnormal electrophysiological properties. Exp. Neurol. 263, 190-199. doi: 10.1016/j. expneurol.2014.10.005

Shalem, O., Sanjana, N. E., Hartenian, E., Shi, X., Scott, D. A., Mikkelson, T., et al. (2014). Genome-scale CRISPR-Cas9 knockout screening in human cells. Science 343, 84-87. doi: 10.1126/science.1247005

Shi, Y., Lin, S., Staats, K. A., Li, Y., Chang, W.-H., Hung, S.-T., et al. (2018). Haploinsufficiency leads to neurodegeneration in C9ORF72 ALS/FTD human induced motor neurons. Nat. Med. 24, 313-325. doi: 10.1038/nm.4490

Song, M., Yang, X., Ren, X., Maliskova, L., Li, B., Jones, I. R., et al. (2019). Mapping cis-regulatory chromatin contacts in neural cells links neuropsychiatric disorder risk variants to target genes. Nat. Genet. 51, 1252-1262. doi: 10.1038/ s41588-019-0472-1

Stone, E. J., Uchida, A., and Brown, A. (2019). Charcot-Marie-Tooth disease Type $2 \mathrm{E} / 1 \mathrm{~F}$ mutant neurofilament proteins assemble into neurofilaments. Cytoskeleton 76, 423-439. doi: 10.1002/cm.21566
Szaro, B. G., and Strong, M. J. (2010). Post-transcriptional control of neurofilaments: new roles in development, regeneration and neurodegenerative disease. Trends Neurosci. 33, 27-37. doi: 10.1016/j.tins.2009.10.002

Timmerman, V., Strickland, A. V., and Züchner, S. (2014). Genetics of CharcotMarie-Tooth (CMT) Disease within the Frame of the Human Genome Project Success. Genes 5, 13-32. doi: 10.3390/genes5010013

Tycko, J., Barrera, L. A., Huston, N. C., Friedland, A. E., Wu, X., Gootenberg, J. S., et al. (2018). Pairwise library screen systematically interrogates Staphylococcus aureus Cas9 specificity in human cells. Nat. Commun. 9:2962. doi: 10.1038/ s41467-018-05391-2

Vakulskas, C. A., Dever, D. P., Rettig, G. R., Turk, R., Jacobi, A. M., Collingwood, M. A., et al. (2018). A high-fidelity Cas 9 mutant delivered as a ribonucleoprotein complex enables efficient gene editing in human hematopoietic stem and progenitor cells. Nat. Med. 24, 1216-1224. doi: 10.1038/s41591-018-0137-0

Watry, H. L., Feliciano, C. M., Gjoni, K., Takahashi, G., Miyaoka, Y., Conklin, B. R., et al. (2020). Rapid, precise quantification of large DNA excisions and inversions by ddPCR. Sci. Rep. 10:14896. doi: 10.1038/s41598-020-71742-z

Wienert, B., Wyman, S. K., Richardson, C. D., Yeh, C. D., Akcakaya, P., Porritt, M. J., et al. (2019). Unbiased detection of CRISPR off-targets in vivo using DISCOVER-Seq. Science 364, 286-289. doi: 10.1126/science.aav9023

Yang, Y., Gu, L.-Q., Burnette, W. B., and Li, J. (2016). N98S mutation in NEFL gene is dominantly inherited with a phenotype of polyneuropathy and cerebellar atrophy. J. Neurol. Sci. 365, 46-47. doi: 10.1016/j.jns.2016.04.007

Yuan, A., Rao, M. V., Veeranna, and Nixon, R. A. (2017). Neurofilaments and neurofilament proteins in health and disease. Cold Spring Harb. Perspect. Biol. 9:a018309. doi: 10.1101/cshperspect.a018309

Yum, S. W., Zhang, J., Mo, K., Li, J., and Scherer, S. S. (2009). A novel recessive Nefl mutation causes a severe, early-onset axonal neuropathy. Ann. Neurol. 66, 759-770. doi: 10.1002/ana.21728

Yu-Wai-Man, P. (2016). Genetic manipulation for inherited neurodegenerative diseases: myth or reality? Br. J. Ophthalmol. 100, 1322-1331. doi: 10.1136/ bjophthalmol-2015-308329

Zaleta-Rivera, K., Dainis, A., Ribeiro, A. J. S., Cordero, P., Rubio, G., Shang, C., et al. (2019). Allele-specific silencing ameliorates restrictive cardiomyopathy attributable to a human myosin regulatory light chain mutation. Circulation 140, 765-778. doi: 10.1161/CIRCULATIONAHA.118.036965

Zhao, J., Brown, K., and Liem, R. K. H. (2017). Abnormal neurofilament inclusions and segregations in dorsal root ganglia of a Charcot-Marie-Tooth type 2E mouse model. PLoS One 12:e0180038. doi: 10.1371/journal.pone.01 80038

Conflict of Interest: $\mathrm{BC}$ is a founder of Tenaya Therapeutics (https: //www.tenayatherapeutics.com/), a company focused on finding treatments for heart failure, including genetic cardiomyopathies. BC holds equity in Tenaya, and Tenaya provides research support for heart failure related research.

The remaining authors declare that the research was conducted in the absence of any commercial or financial relationships that could be construed as a potential conflict of interest.

Publisher's Note: All claims expressed in this article are solely those of the authors and do not necessarily represent those of their affiliated organizations, or those of the publisher, the editors and the reviewers. Any product that may be evaluated in this article, or claim that may be made by its manufacturer, is not guaranteed or endorsed by the publisher.

Copyright (c) 2021 Feliciano, Wu, Watry, Marley, Ramadoss, Ghanim, Liu, Zholudeva, McDevitt, Saporta, Conklin and Judge. This is an open-access article distributed under the terms of the Creative Commons Attribution License (CC BY). The use, distribution or reproduction in other forums is permitted, provided the original author(s) and the copyright owner(s) are credited and that the original publication in this journal is cited, in accordance with accepted academic practice. No use, distribution or reproduction is permitted which does not comply with these terms. 